\title{
Enfisema Lobar Congénito, reporte de dos casos
}

Alvaro Mallea Pérez', Aylin Aliaga Reguerin', Alberto Morató López ${ }^{2}$

\section{RESUMEN}

El enfisema lobar congénito es una entidad poco común. No existe una etiología clara y se ha descrito que la causa más común es la displasia del cartílago bronquial. El diagnóstico clínico es difícil de realizar en tanto que la tomografía multidetector, así como el estudio histopatológico pueden definir el diagnóstico. Presentamos los casos clínicos de dos neonatos de sexo masculino con clínica similar a la de una neumonía. Describimos el proceso con el cual llegamos a la conclusión diagnóstica, su manejo, pronóstico y una breve revisión de la literatura. (Horiz Med 2014; 14(2): 53-57)

Palabras clave: Enfisema lobar congénito, tomografía multidetector, neumonía.(Fuente: DeCS BIREME).

\section{Congenital Lobar Emphysema, Two cases report}

\begin{abstract}
Congenital lobar emphysema is a rare entity; there is no clear etiology and it has been described that the most common cause is the bronchial cartilage dysplasia. Clinical diagnosis is difficult to perform and multidetector computed tomography either the histopathological study can define the diagnosis. We present two cases of two male neonates with similar symptomatology than pneumonia. We describe the process that lead us to the diagnostic conclusion, management, prognostic and we present a brief review of the literature.
\end{abstract}

(Horiz Med 2014; 14(2): 53-57)

Key words: Congenital lobar emphysema, CT scan, pneumonia.(Source: MeSH NLM). 


\section{INTRODUCCIÓN}

El término enfisema lobar congénito (ELC) fue usado por Robertson y James en 1951 para denotar al enfisema infantil cuando procesos como la infección pulmonar o los cuerpos extraños intrabronquiales se encontrasen ausentes.

Las enfermedades respiratorias, son una de las causas más comunes de mortalidad en Unidad de cuidados intensivos neonatales (UCIN). Están divididas en dos grupos mayores: adquiridos y congénitos. Los síndromes que presentan alteración del desarrollo pulmonar congénito, se manifiestan con hipertensión pulmonar primaria y dificultad respiratoria $(1,2)$.

El ELC, es una entidad rara que se presenta en los primeros meses de vida, es más común en varones que en mujeres y la incidencia reportada es entre 1 en 70.000 a 1 en 90.000 nacidos vivos (3).

Usualmente, solo un lóbulo pulmonar está involucrado, sin embargo; al gunos pacientes pueden presentar un involucro lobar múltiple. Afectando de forma usual al lóbulo superior izquierdo $(43 \%)$ seguido por el lóbulo medio derecho $(32 \%)(4,5)$. De las posibles causas, podemos hallar factores intrínsecos o extrínsecos.

Factores intrínsecos: displasia del cartílago bronquial, estenosis bronquial, broncomalacia, vasos sanguíneos aberrantes o mucosa bronquial redundante.

Factores extrínsecos: compresión bronquial por anillos vasculares o linfonodos $(6,7)$. Se ha reportado que el ELC también esta asociado a malformaciones cardiacas congénitas, dentro de las cuales podemos citar a la hipertensión pulmonar, Tetralogía de Fallot y persistencia del conducto arterioso (7-9).

Estudios documentados, indican que el $25 \%$ de los casos de ELC se deben a la displasia del cartílago bronquial $(4,10)$. La teoría más reciente propone un aumento en el número de alveolos dentro de cada acíno (lóbulo polialveolar) (11).

Clínicamente, el ELC se caracteriza por presentar diferentes grados de dificultad respiratoria, evidencia clínica y radiológica de hiperinsuflación de uno o mas lóbulos del pulmón lo cual resulta en atelectasia por compresión, lateralización mediastinal, hipoxia e hipotensión (12).

El diagnóstico de ELC puede alcanzarse mediante una combinación de características clínicas, radiológicas e histopatológicas y el manejo no es diferente al de otras patologías respiratorias.

Otras entidades patológicas con la cual habitualmente existe confusión, es con neumotórax y la neumonía $(13,14)$.

En casos en los que el lóbulo enfisematoso es grande, la herniación del pulmón al hemitórax contralateral y atelectasia del pulmón contralateral pueden ser observados $(10,11,15)$.

La Tomografía computarizada, representa una modalidad de diagnóstico no invasiva que demuestra la anatomía en múltiples proyecciones gracias a su capacidad de reconstrucción multiplanar que permite evaluar la arquitectura parenquimatosa pulmonar, las acarteristicas anatomicas del arbol traqueo-bronquial, la local ización y extensión exacta del defecto y anomalías acompañantes (16-18). A la vez permite descartar alternativas diagnósticas al ELC como cuerpo extraño bronquial (19).

En situaciones dudosas la fluoroscopía, broncoscopía, angiografía, evaluación radioisotópica y escintografía pulmonar pueden ser también de ayuda $(11,16,17)$.

El tratamiento es principalmente quirúrgico con muy pocos índices de mortalidad (18).

\section{CASO CLÍNICO 1}

RN de sexo masculino, transferido de un Centro Hospitalario de la ciudad de El Alto. Producto de segundo embarazo, parto por cesárea, Apgar 9-10, $3.900 \mathrm{gr}$ al nacimiento. Sin antecedentes perinatales ni familiares de importancia. El que fue externado de dicho centro hospitalario a las 24 horas y dado de altaa las 72 horas.

La madre refirió, que el neonato presentó dificultad respiratoria y alteración del estado general. Fue reevaluado e ingresado con el diagnóstico de neumonía y posible sepsis. 
Se tomó radiografía de tórax: signos de patología pulmonar que reforzó la sospecha clínica. Laboratorio: biometría hemática, PCR y gasometría arterialdentro de parámetros normales. Se inició antibiótico terapia sin respuesta alguna, al no disponer de (UCIN) deciden transferencia.

Nuevamente es valorado en el Hospital Juan XXIII, evidenciando taquipnea, retracción intercostal, aleteo nasal con compromiso del estado general. Se continuó con antibiótico terapia y se inició CPAP nasal en UCIN. Al no hallarse respuesta, se solicita TC de tórax simple:hiperinsuflación con pérdida de la arquitectura parenquimatosa del lóbulo superior derecho con enfisema centrolobulillar y panlobulillar que condicionaba efecto de desplazamiento severo con desplazamiento mediastínico.

Los lóbulos pulmonares medio e inferior derecho, así como el lóbulo inferior izquierdo mostraron incremento de la densidad con patrón en vidrio despulido con probable relación a colapso alveolar parcial de tipo relajativo, sin consolidación franca. En el lóbulo inferior izquierdo se observó una opacidad triangular de vértice hiliar sin broncograma compatible con atelectasia obstructiva. Figuras 1 y 2.

Debido a ello, el paciente es sometido a procedimiento quirúrgico: lobectomía superior derecha y llevado a UCIN para manejo postoperatorio donde permaneció 15 días dándosele de alta en buen estado general para control por consultorio externo.

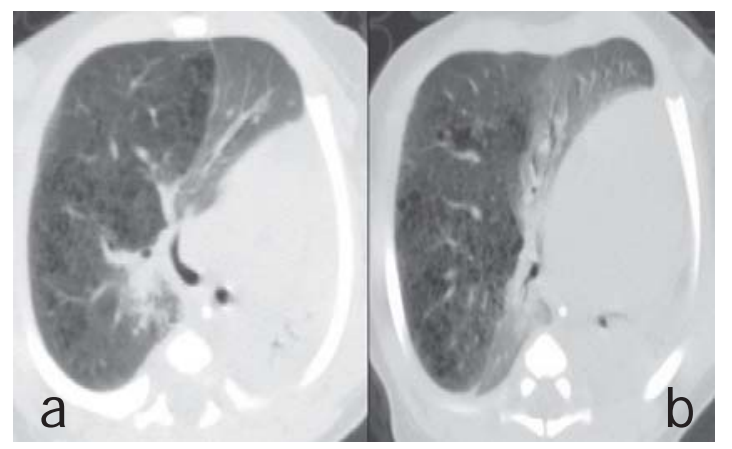

Figura 1. Caso 1. Imágenes de TC. en proyección axial y con ventana de parénquima pulmonar. Se muestra hipodensidad parenquimatosa del lóbulo medio con imágenes redondeadas de menor densidad que corresponden a bulas enfisematosas que traducen ruptura de las paredes alveolares. Hiperinsuflación secundaria que condiciona desplazamiento izquierdo del mediastino, atelectasia por relajación del resto del parénquima pulmonar

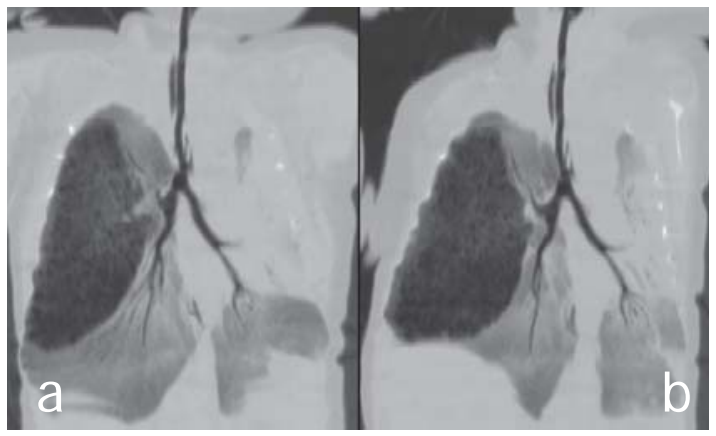

Figura 2.- Caso 1. Reconstrucciones multiplanares de TC en proyección coronal, demostrando el árbol traqueobronquial. Se delimita claramente el enfisema lobar medio con delimitación cisural, pérdida de la arquitectura e hiperinsuflación. No se visualiza el bronquio del lóbulo medio (está hipotrófico). En el pulmón izquierdo hay opacificación pulmonar con agrupamiento bronquial que traduce atelectasia por relación, una de ellas con morfología triangular sin broncograma, que puede estar en relación a atelectasia obstructiva.

\section{CASO CLÍNICO 2}

RN de 2 horas de vida, de sexo masculino. Residente de la ciudad de La Paz transferido de otro centro hospitalario con diagnóstico de sepsis.

Ingresado a UCIN con dificultad respiratoria. Al examen físico: paciente afebril, tiraje marcado, aleteo nasal, sibilancias, disminución del murmullo vesicular, matidez a la percusión. SatO2 76\%, frecuencia respiratoria $58 \times 1^{\prime}$, frecuencia cardiaca en $118 \times 1$ 'y presencia de cianosis central y periférica. Se inició antibióticoterapia profiláctica, se tomaron muestras para cultivos, biometría hemática, gasometría y electrolitos así como radiografía de tórax simple. Al no evidenciarse grandes cambios laboratoriales y al presenciar cambios sugestivos de neumonía, se decidió realizar tomografía de tórax en base a la experiencia pasada, hallándose:hipodensidad del lóbulo medio con lesiones enfisematosas centrolobulillares casi imperceptibles.

Con afección del lóbulo inferior izquierdo en menor gravedad y leve efecto de volúmen con desplazamiento mediastinal. Figura 3.

Fue sometido a lobectomía con buenos resultados. La muestra histopatológica concordó con el diagnóstico de enfisema lobar congénito. Permaneciendo en internación durante 17 días, se le dió de alta en buenas condiciones. 


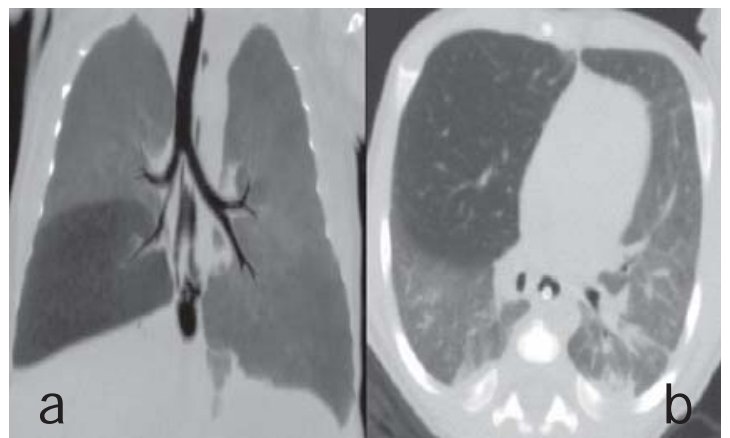

Figura 3. Caso 2. (a) Imagen de TC en proyección axial, se demuestra hipodensidad del lóbulo medio con lesiones enfisematosas centrilobulillares casi imperceptibles. El lóbulo inferior izquierdo también esta afectado pero en menor gravedad. Existe leve efecto de volumen con desplazamiento mediastinal. (b) reconstrucción multiplanar en proyección coronal que demuestra claramente el árbol traqueobronquial con calibre reducido del bronquio interlobar, y el área hiperinsuflada y de menor densidad delpulmón derecho.

\section{DISCUSIÓN}

El enfisema lobar congénito es poco frecuente, por lo que merece atención especializada para llegar a su diagnóstico. La identificación temprana es la base fundamental para lograr un abordaje oportuno. Lamentablemente, los datos clínicos son similares a muchos procesos respiratorios agudos y la radiografia de tórax frecuentemente no es concluyente (4).

Se requiere de estudios avanzados, lo que es una limitación en nuestro país. La evidencia enmarca que ciertos datos clínicos como la falta de respuesta ante la terapéutica común puede ser un primer punto diana para llegar a una resolución diagnóstica evitando así procedimientos innecesarios que pueden empeorar el cuadro clínico.

La poca incidencia y prevalencia de casos de ésta magnitud nos lleva a afinar el juicio clínico y poder optimizar los recursos del estado para tener un equipo multidisciplinario preparado para afrontar este tipo de situaciones ya que el tratamiento quirúrgico y los cuidados postoperatorios pueden resolver el problema, prolongando y mejorando la sobrevida de este grupo de pacientes.

La TC multidetector, es un método de diagnóstico por imágenes rápido, versátil y de alta confiabilidad, que proporciona alto detalle anatómico con capacidad de representación multiplanar. Permite reunir información que puede ser concluyente para establecer diagnósticos como el ELC y evaluar diagnósticos alternos como otras anomalias congénitas del tórax, descarte de cuerpos extraños intrabronquiales, y consecuentemente orientar la estrategia terapéutica de manera oportuna (18).

\section{Fuente de Financiamiento}

El presente trabajo fue financiado a través de recursos propios de los investigadores.

\section{Conflictos de interés}

Los autores declaran no tener conflictos de interés en la publicación de este artículo.

\section{REFERENCIAS BIBLIOGRÁFICAS}

1. Behrman R \& Kliegman R. Nelson Textbook of Pediatrics, Vol 2, 19th Ed, W.B.Saunders Elsevier, Philadelphia, 2010.

2. Paramesh H. Textbook of Practical Pediatric Pulmonology. 1st ed. New Delhi, Jayppe Brothers Medical Pub, 2009.

3. Ward CF. Diseases of infants. Anesthesia and uncommon diseases. In: Katz J, Benumof JL, Kadis LB editors. Philadelphia: W.B. Saunders Co; 1990.

4. Al-Salem AH, Gyamfi YA, Grant CS. Congenital lobar emphysema. Can J Anaesth 1990;37:377-9.

5. Ozçelik U, Göçmen A, Kiper N, Doğru D, Dilber E, Yalçin EG. Congenital lobar emphysema: evaluation and long- term follow up of thirty cases at a single centre. Pediar Pulmonol 2003;35:384-91.

6. Karapurkar SA, Borkar JD, Birmole BJ. Malformation of lung in neonates (lobectomy for congenital lobar emphysema and lung cyst). J Postgrad Med 1993;39:224.

7. Moideen I, Nair SG, Cherian A, Rao SG. Congenital lobar emphysema associated with congenital heart disease. J Cardio thorac Vasc Anesth 2006;20:239-41.

8. Hishitani T, Ogawa K, Hoshino K, et al. Lobar emphysema due to ductus arteriosus compressing right upper bronchus in an infant with congenital heart disease. Ann Thorac Surg 2003;75:1308-10.

9. Isojima A, Yuasa $H$, Kusagawa $M$, et al. Surgical treatment of infantile lobar emphysema in cardiovascular disease with left-toright shunts.Jpn J Surg 1978;8:57-65.

10. Franken EA, Buehl I. Infantile lobar emphysema: report of two cases with unusual roentgenographic manifestations. Am J Roentgenol 1966;98:354-7. 
11. Guidici R, Leao LE, Moura LA, Wey SB, Ferreira RG, Crotti PL. Polyalveolosis: pathogenesis of congenital lobar emphysema? Rev Assoc Med Bras 1998; 44:99-105.

12. Cote CJ. The anesthetic management of congenital lobar emphysema. Anesthesiology 1978;19: 296-8.

13. Man D, Hamdy MH, Hendry GM, Bisset WH, Forfar JO. Congenital lobar emphysema: problems in diagnosis and management. Arch Dis Child 1983;58:709-12.

14. Karnak I, Senocak ME, Ciftci AO, Büyükpamukçu N. Congenital lobar emphysema: diagnostic and therapeutic considerations. J Pediatr Surg 1999;34: 1347-51.

15. Gupta R, Singhal SK, Rattan KN, Chhabra B. Management of congenital lobar emphysema with endobronchial intubation and controlled ventilation. Anesth Analg 1998;86: 71-3.

16. Mandelbaum I, Heimburger I, Battersby JS. Congenital lobar obstructive emphysema: report of eight cases and literature review. Ann Surg 1965;162:1075-80

17. Loewy L, O’Brodowich H, Coates G. Ventilation scintigraphy with submicrinic radioaersol as adjunct in the diagnosis of congenital lobar emphysema. J Nucl Med 1987;28:1213-7.

18. Al-Salem AH. Congenital lobar emphysema. Saudi Med J 2002;23:335.

19. Song Z, Ge W, Xu C, Fang B, Yang C, Zhang H, Zhao Y. The diagnostic value of multislice computed tomography in children with congenital lobar emphysema. Expert Rev Med Devices 2011; 8: 435-9.

20. Hochhegger B, Klaus L, Feijó C, Zanetti G, Marchiori E. Congenital lobar emphysema: the role of multislice computed tomography with virtual broncoscopy in the differential diagnosis with bronchial foreing bodies. Eu Arch of Otorhinolaryngol 2012; 269: 2015-6.

\section{Correspondencia:}

Alvaro Mallea Pérez

Dirección: Av. Naciones Unidas s/n, La Paz-Bolivia

Teléfono: +59 176212285

Correo electrónico: alvaro_mape@hotmail.com

Recibido: 03 de Diciembre de 2013 Aprobado: 17 de Abril de 2014 